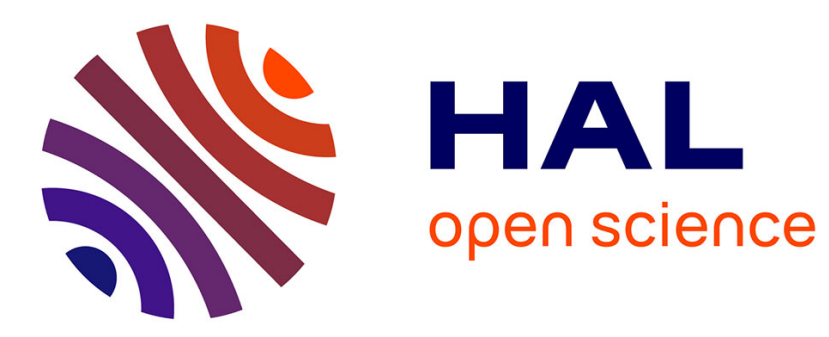

\title{
Time Scale Approach for Chirp Detection
}

\author{
Marcela Morvidone, Bruno Torrésani
}

\section{To cite this version:}

Marcela Morvidone, Bruno Torrésani. Time Scale Approach for Chirp Detection. International Journal of Wavelets, Multiresolution and Information Processing, 2003, 1 (1), pp.19-50. 10.1142/S0219691303000037 . hal-01278587

\section{HAL Id: hal-01278587 \\ https://hal.science/hal-01278587}

Submitted on 24 Feb 2016

HAL is a multi-disciplinary open access archive for the deposit and dissemination of scientific research documents, whether they are published or not. The documents may come from teaching and research institutions in France or abroad, or from public or private research centers.
L'archive ouverte pluridisciplinaire $\mathbf{H A L}$, est destinée au dépôt et à la diffusion de documents scientifiques de niveau recherche, publiés ou non, émanant des établissements d'enseignement et de recherche français ou étrangers, des laboratoires publics ou privés. 


\title{
TIME SCALE APPROACH FOR CHIRP DETECTION
}

\author{
Marcela MORVIDONE \\ Laboratoire d'Analyse, Topologie et Probabilités, Université de Provence, Centre de \\ Mathématiques et Informatique, 31 rue Joliot-Curie \\ 13351 Marseille Cedex 13, France. \\ morvidon@cmi.univ-mrs.fr \\ Bruno TORRESANI \\ Laboratoire d'Analyse, Topologie et Probabilités, Université de Provence, Centre de \\ Mathématiques et Informatique, 31 rue Joliot-Curie \\ 13351 Marseille Cedex 13, France. \\ Bruno.Torresani@cmi.univ-mrs.fr \\ Received (1 November 2002)
}

\begin{abstract}
Two different approaches for joint detection and estimation of signals embedded in stationary random noise are considered and compared, for the subclass of amplitude and frequency modulated signals. Matched filter approaches are compared to time-frequency and time scale based approaches. Particular attention is paid to the case of the so-called "power-law chirps", characterized by monomial and polynomial amplitude and frequency functions. As target application, the problem of gravitational waves at interferometric detectors is considered.
\end{abstract}

Keywords: signal detection/estimation; cross ambiguity function; wavelet transform; ridge detection.

AMS Subject Classification: 41A60, 42C40, 60G35, 62P35

\section{Introduction}

The problem of joint detection and estimation of parametrized signals embedded in stationary random noise is generally considered a difficult one, since it combines two difficulties. Traditional approaches often rely on variations around generalized correlations and ambiguity functions. More specifically, a generalized "cross ambiguity function" between the observed signal and templates of the expected signals, and the maxima of this ambiguity function which are above some threshold yield candidate "events". However, such approaches may happen to lack of robustness and/or become particularly expensive (in terms of computational cost) for some classes of signals. This is mainly due to the fact that for some signal classes, ambiguity functions may feature sharp variations, which yield either a lack of robustness or high computational costs. In such situations, one may turn to alternatives, which are generally suboptimal in terms of detection (i.e yield higher non-detection or false 
alarm probabilities), but turn out to be more robust or computationally simpler.

In this paper, we focus on the particular case of amplitude and frequency modulated signals, and study an alternative approach based on the continuous wavelet transform. It may be shown that the continuous wavelet transform of such signals generally enjoys sharp localization properties in the time-scale plane, which may be exploited in a suitable detection algorithm. The latter may be expected to underperform the ambiguity function approach in terms of pure detection (false alarm or missed detections), but also to behave better in terms of robustness and computational burden.

The main idea we follow here consists in seeking optimal curves in the time-scale plane, i.e. curves which optimize some "time-scale energy". The optimal curves are searched within a parametric family, the parameters being put into correspondence with the instantaneous frequency of the seeked signal. The so-obtained energy function turns out to have much slower variations than the ambiguity functions, which simplifies the detection procedure. However, such simplifications also yield a lowering of the detection performances of the algorithm. It is one of the goals of this paper to study this tradeoff.

As target application, we consider the problem of detection/estimation of gravitational waves at interferometric detectors. The existence of such waves was predicted long ago by general relativity, but no direct observation could be done so far. Large scale experiments are currently being prepared (among which the VIRGO ${ }^{2}$, LIGO$^{1}$ and TAMA projects), which should be able to provide such direct observations. Among the potential sources for gravity waves, the waves generated by coalescing binary star systems are particularly appealing, since the corresponding waveform is fairly well-known, and may be given (to some approximation degree) an explicit expression. It takes the form of a power law chirp (i.e. a frequency and amplitude modulated signal, with power law instantaneous frequency), embedded into stationary random noise, with prescribed spectral density. The two approaches described above may be adapted to this context. We present the general theory associated to the detection of such "power law chirps" using generalized cross-ambiguity functions (i.e. banks of matched filters) and continuous wavelet transform line integrals, and discuss corresponding numerical results. The first results on comparison of the two approaches in terms of detection performances are also given.

The present paper is organized as follows. We first discuss (Section 2) in some details the problem of joint signal detection and estimation, and introduce the gereralized cross ambiguity function as the optimal solution to this problem. We also consider the class of amplitude and frequency modulated signals (the so-called chirps), and provide estimates (using oscillatory integrals approximation techniques) for the behavior of the ambiguity function for such signal classes. We then describe and study in Section 3 the proposed alternative approach, based upon wavelet transform ridge detection, and provide indications about its behavior for the considered signals. We also provide estimates for the behavior of the continuous wavelet trans- 
form of the considered signal. We then turn to the application to gravitational waves detection in Section 4, and compare the performances of the two approaches on simulated gravitational signals. The numerical results obtained in the absence of noise provide numerical confirmations for the approximations obtained in the previous sections, and show the relevance of the approaches under consideration. Numerical simulations for noisy signals in physically realistic situations are also presented, which illustrate the domain values of parameters within which the wavelet transform line integral approach may be considered a suitable alternative to the ambiguity function approach. Some more mathematical aspects (in particular, proofs of some estimates) are also discussed in appendices.

\section{Joint detection/estimation, chirps and cross-ambiguity functions}

\subsection{Problem statement}

We consider the following joint detection-estimation problem: Let a parametric family of signals $\left\{x_{\theta}, \theta \in \Theta\right\}$ be given ${ }^{\mathrm{a}}, \theta \in \Theta$ being a multidimensional parameter. Assume that we are given observations of the form

$$
\begin{aligned}
& H_{0}: y(t)=X_{t}, \\
& H_{1}: y(t)=A x_{\theta}(t)+X_{t},
\end{aligned}
$$

where $A \in \mathbb{R}^{+}$is an (unknown) multiplicative constant, and $X$ is a noise, modeled as a gaussian random process. The considered problem involves simultaneously hypothesis testing (decide whether the signal is present or not) and parameter estimation (under the $H_{1}$ hypothesis, estimate the vector parameter $\theta \in \Theta$ ).

In this paper, we shall always assume that the noise $X$ is modelled as a gaussian second order random process, second order continuous and stationary. ${ }^{15,16} \mathrm{We}$ assume further that its spectral measure $d \nu_{X}$ is absolutely continuous. As a result, the noise process $X$ is completely characterized by its power spectral density $\mathscr{S}_{X}$ : its covariance function $C_{X}$ satisfies

$$
C_{X}(t, s)=\mathbb{E}\left\{X_{t} \bar{X}_{s}\right\}=C_{X}(t-s)=\int_{-\infty}^{\infty} e^{2 i \pi \nu(t-s)} \mathscr{S}_{X}(\nu) d \nu
$$

The corresponding covariance operator will be denoted by $\mathscr{C}_{X}$ :

$$
\mathscr{C}_{X} f(t)=\int_{-\infty}^{\infty} C_{X}(t-s) f(s) d s,
$$

and we introduce

$$
\mathscr{E}_{X}=\left\{x \in L^{2}(\mathbb{R}), \mathscr{C}_{X}^{-1 / 2} x \in L^{2}(\mathbb{R})\right\}
$$

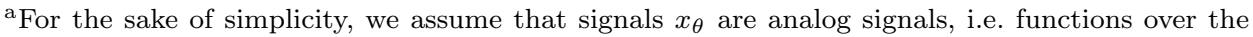
real line. The present discussion may easily be adapted to numeric signals, or signals defined on bounded intervals, after suitable choice of boundary conditions. 
A traditional approach typically makes use of the so-called cross-ambiguity function.

Definition 2.1. Let $\left\{x_{\theta} \in \mathscr{E}_{X}, \theta \in \Theta\right\}$ be a parametric class of finite energy signals, assumed to be embedded in zero mean, second order continuous and stationary random noise with spectral density $\mathscr{S}_{X}$. Given a signal $f \in \mathscr{E}_{X}$, the corresponding generalized ambiguity function ${ }^{\mathrm{b}}$ is defined by the mapping

$$
\theta \rightarrow \mathscr{A}_{f}^{(n)}(\theta)=\frac{\left\langle f, \mathscr{C}_{X}^{-1} x_{\theta}\right\rangle}{\sqrt{\left\langle x_{\theta}, \mathscr{C}_{X}^{-1} x_{\theta}\right\rangle}} .
$$

Coming back to the signal model (2.1) we have, under the $H_{1}$ hypothesis

$$
\mathscr{A}_{y}^{(n)}(\theta)=A \mathscr{A}_{x_{\theta_{0}}}^{(n)}(\theta)+N(\theta) .
$$

The first term is the output signal and the second is the output noise. Considering the "signal" term, the following statement is a direct consequence of the Schwarz inequality.

Proposition 2.1. Under the assumptions of Definition 2.1, for $\theta_{0} \in \Theta$ and for all $\theta \in \Theta$ such that $x_{\theta}$ and $x_{\theta_{0}}$ are linearly independent,

$$
\left|\mathscr{A}_{x_{\theta_{0}}}^{(n)}(\theta)\right| \leq \mathscr{A}_{x_{\theta_{0}}}^{(n)}\left(\theta_{0}\right)
$$

with equality if and only if $\theta=\theta_{0}$.

The noise term is a gaussian random function of $\theta$. Clearly, $\mathbb{E}\{N(\theta)\}=0$, and $\operatorname{Var}(N(\theta))=1$. Hence, the optimal $S N R^{\mathrm{c}}$ is given by

$$
\rho=\frac{\mathscr{A}_{x_{0}}^{(n)}\left(\theta_{0}\right)}{\sqrt{\operatorname{Var}(N(\theta))}}=\mathscr{A}_{x_{\theta_{0}}}^{(n)}\left(\theta_{0}\right)=\left\|\mathscr{C}_{X}^{-1 / 2} x_{\theta_{0}}\right\| .
$$

This result is the basis of the usual detection-estimation algorithms: given an observed signal $y$, the following operations are performed

(i) Compute the values of the generalized cross-ambiguity function $\mathscr{A}_{y}^{(n)}(\theta)$, for a (suitably sampled) set of values for the parameters $\theta$.

(ii) The values of $\theta$ such that $\mathscr{A}_{y}^{(n)}(\theta)$ exceeds a fixed threshold are candidates for possible "events".

Remark 2.1. Clearly enough, the "speed" of variation of the ambiguity function plays a key role in the detection problem. Rapidly varying functions $\mathscr{A}^{(n)}$ require a dense sampling of the parameter set, and thus yields high computational costs. Conversely, an unsufficiently dense sampling of the parameter set increases the risk of "missing the maximal value" of $\mathscr{A}^{(n)}$, and lowers the performances of the algorithm.

\footnotetext{
b the superscript " $(n)$ " stands for "normalized"

"SNR stands for "Signal to Noise Ratio".
} 
Remark 2.2. The choice of the threshold is another important issue: it controls the rate of false alarms (values of $\mathscr{A}^{(n)}$ larger than the threshold due to noise), and missed detections (situations where $\mathscr{A}^{(n)}(\theta)$ is smaller than the threshold, despite of the presence of signal, again because of noise). Practical strategies consist in adapting the threshold to a fixed admissible false alarm probability, or a fixed admissible non-detection probability. ${ }^{24}$

Remark 2.3. The amplitude parameter $A$ in (2.1) is also to be estimated from the observations. Clearly, once a signal with parameters $\theta_{0}$ has been detected, a natural estimate for $A$ is given by $\hat{A}=\mathscr{A}_{y}^{(n)}\left(\theta_{0}\right) / \rho$. However, too small values of $A$ make detection impossible. The detectability threshold, i.e. the smallest $A$ such that detection is considered possible, depends on the optimal SNR $\rho$.

\subsection{Chirps}

We are concerned in this paper with signal classes formed of chirp signals. A chirp is essentially a frequency and amplitude modulated signal, of the form

$$
x(t)=a(t) \cos (\varphi(t)),
$$

and additional assumptions on the relative speed of variations of the local amplitude $a$ and frequency $\varphi^{\prime}$ are generally made to ensure that the latter quantities have physical meaning. In such situations, it is customary to work with the so-called analytic signals, associated with such signals, and the corresponding instantaneous amplitude and frequency:

Definition 2.2. Let $x \in L^{2}(\mathbb{R})$ be a finite-energy signal. The associated analytic signal $z_{x} \in L^{2}(\mathbb{R})$ is defined by its Fourier transform

$$
z_{x}(t)=2 \int_{0}^{\infty} e^{2 i \pi \nu t} \hat{x}(\nu) d \nu
$$

The canonical pair $\left(A_{x}, \phi_{x}\right)$ associated with $x$ is then defined by

$$
A_{x}=\left|z_{x}\right|, \quad \phi_{x}=\arg \left(z_{x}\right) .
$$

$A_{x}$ and $\phi_{x}$ are termed respectively instantaneous amplitude and instantaneous phase, and $\phi_{x}^{\prime}$ is the instantaneous frequency.

It is worth mentioning that the notions of local amplitude $a$ and frequency $\varphi$ which appear in (2.5) and instantaneous amplitude and frequency as in Definition 2.2 do not coincide in general ${ }^{9,21}$ (in such situations, the physical meaning of such quantities may however become questionable.) Nevertheless, under some additional assumptions, they may be shown to be fairly close to each other, as shown by the following result, whose proof may be found in Appendix A.

Proposition 2.2. Let $x$ be a chirp signal as in (2.5), such that $a \in C^{1}$ has bounded support and vanishes at boundaries, and $\varphi \in C^{2}$. Assume further that the local frequency is bounded from below: $\varphi^{\prime}>K$ for some $K \in \mathbb{R}^{+}$. Then

$$
z_{x}(t)=a(t) e^{i \varphi(t)}+r(t),
$$


where

$$
\|r\|_{\infty} \leq\|a\|_{1}\left(\frac{\left\|a^{\prime}\right\|_{1}}{\|a\|_{1}}\left\|\frac{1}{\varphi^{\prime}}\right\|_{\infty}+\left\|\frac{\varphi^{\prime \prime}}{\varphi^{\prime 2}}\right\|_{\infty}\right) .
$$

This simply shows that if the local amplitude and frequency vary slowly enough on the support of the signal, the analytic signal is close to the "exponential model" $a \exp (i \varphi)$. Results in the same spirit may also be obtained in the case of signals with infinite support, using a partition of unity.

In this work, we shall often make use of the Fourier transforms of chirp signals. The stationary phase approximations ${ }^{11}$ provide a way to estimate such Fourier transforms. Such approximations turn out to be extremely precise for very oscillatory signals. The proof of the following result may be found in Appendix A

Proposition 2.3. Let $x=a \exp (i \varphi)$, where $a \in C^{2}$ is a positive-valued function, and $\varphi \in C^{5}$ is such that $\varphi^{\prime}>K>0$ for some constant $K$. Assume further that $\varphi^{\prime}$ is monotonic. Then, for all $\nu \in \operatorname{range}\left(\varphi^{\prime}\right) / 2 \pi$, we have

$$
\hat{x}(\nu)=\sqrt{2 \pi} \frac{a\left(t_{s}\right)}{\sqrt{\left|\varphi^{\prime \prime}\left(t_{s}\right)\right|}} e^{i\left(\varphi\left(t_{s}\right)-2 \pi \nu t_{s}\right)} e^{i s g n\left(\varphi^{\prime \prime}\left(t_{s}\right)\right) \pi / 4}+r(\nu),
$$

where $t_{s}=t_{s}(\nu)$ is the (unique) solution of

$$
\varphi^{\prime}\left(t_{s}\right)=2 \pi \nu .
$$

The remainder $r$ depends upon the derivatives of $a$ and $\varphi$ up to degrees two and three respectively.

A precise bound for the remainder may be found in Appendix A.

Particular attention has recently been paid to the class of so-called "power-law chirps" 18 . The latter are characterized by the power law behavior of their local amplitude and phase functions, of the form

$$
x(t)=\left(t_{0}-t\right)^{\alpha} \cos \left(\frac{2 \pi F_{0}}{\beta+1}\left(t_{0}-t\right)^{\beta+1}\right) H\left(t_{0}-t\right)
$$

(where $t_{0}$ is a reference "time", and $F_{0}$ is a reference "frequency" , and $H$ denotes Heaviside's step function), to which a local frequency

$$
\nu(t)=F_{0}\left(t_{0}-t\right)^{\beta}
$$

may be associated. $\alpha$ and $\beta$ are typically negative numbers, so that the local amplitude and frequency functions diverge as $t \rightarrow t_{0}$. An example of such power law chirp may be found in Fig 1 below. An immediate consequence of proposition 2.3 above is the approximate expression of the Fourier transform of such power law chirps.

\footnotetext{
deven though it does not have the physical dimension of a frequency.
} 
Corollary 2.1. Let $x$ be a power law chirp as in (2.11). Then its Fourier transform is given by

$$
\hat{x}(\nu)=\hat{x}_{0}(\nu)+r(\nu),
$$

where the leading term reads

$$
\hat{x}_{0}(\nu)=\frac{1}{\sqrt{F_{0}|\beta|}}\left(\frac{\nu}{F_{0}}\right)^{(2 \alpha-\beta+1) / 2 \beta} \exp \left\{2 i \pi\left(-\nu t_{0}+F_{0}^{-1 / \beta} \frac{\beta}{\beta+1} \nu^{(\beta+1) / \beta}+\frac{1}{8}\right)\right\}
$$

and the remainder $r$ behaves as in Proposition 2.3.

The validity of such approximations turn out to be quite questionable, at least for some ranges of values of $\nu$. In fact, the validity domain happens to depend on the values of $\alpha$ and $\beta$. More precisely, it is shown in Appendix A that for $\beta>-1$, such approximations are to be considered as low frequency approximations rather than high frequency ones. ${ }^{4,5}$ For $\beta \leq-1$, such chirps fall into the class of the infinitely oscillating functions studied in great details by Meyer and collaborators. ${ }^{18}$

\subsection{Ambiguity function for power law chirp detection}

In the case of power law chirp signals, the ambiguity function may be given fairly simple approximations, which help understanding its behavior (even in more complex situations).

In this section, we shall take as signal model the Fourier representation provided by the stationary phase approximation, as in equation (2.14). As mentioned earlier, the precision of such approximations happen to be quite questionable within some frequency range for some values of the $F_{0}$ parameter. However, we shall limit ourselves to situations in which this model is accurate enough, i.e. for which the frequency domain significantly involved in the ambiguity function is contained in the validity domain of the approximation. This will be the case for the application considered in Section 4.

Let us then consider a reference signal with parameters $t_{0}$ and $F_{0}$ defined in the Fourier domain as in (2.14), copies with the same form and parameters denoted by $t$ and $F$, and study the corresponding generalized cross-ambiguity function. For the sake of simplicity (to avoid heavy notations and formulas), we shall consider the unnormalized version of the ambiguity function

$$
\mathscr{A}_{f}(\theta)=\left\langle f, \mathscr{C}_{X}^{-1} x_{\theta}\right\rangle
$$

rather than the normalized version $\mathscr{A}^{(n)}$ given in Definition 2.1. This doesn't change anything to the detection/estimation procedure, and the translation from one version to another is easily done. It is easily seen that the generalized crossambiguity function $\mathscr{A}_{f}$ takes the form of a correlation between the input signal $f$ and a family of "templates" (in fact rescaled copies of a reference power law chirp $H(t)(-t)^{\alpha} \cos \left(2 \pi(-t)^{\beta+1}\right)$, modified by the action of $\mathscr{C}_{X}^{-1}$.) Hence, the detector 
based upon generalized cross ambiguity function may also be seen as a bank of matched filters.

By Plancherel's theorem, we have

$$
\mathscr{A}_{\left(t_{0}, F_{0}\right)}(t, F)=\int_{0}^{\infty} \overline{\hat{x}_{\left(t_{0}, F_{0}\right)}}(\nu) \hat{x}_{(t, F)}(\nu) \frac{d \nu}{\mathscr{S}_{X}(\nu)} .
$$

For the sake of simplicity, let us first introduce a few notations. We set

$$
\Delta t=t-t_{0}, \quad \Delta F=F^{-1 / \beta}-F_{0}^{-1 / \beta},
$$

and

$$
M(\nu)=\frac{\nu^{(2 \alpha-\beta+1) / \beta}}{\mathscr{S}_{X}(\nu)} .
$$

In what follows, we shall assume that $M \in L^{1}\left(\mathbb{R}^{+}\right)$(such an assumption becomes essentially academic when it comes to practical situations, as will be stressed in Remark 4.1 below).

According to equation (2.14) above, we obtain the following form for the Ambiguity function:

$$
\mathscr{A}_{\left(t_{0}, F_{0}\right)}(t, F) \approx C\left(F_{0}, F\right) \mathscr{I}(\Delta t, \Delta F),
$$

where the $C\left(F_{0}, F\right)$ constant reads

$$
C\left(F_{0}, F\right)=\frac{1}{|\beta| \sqrt{F_{0} F}} \frac{1}{\left(F_{0} F\right)^{(2 \alpha-\beta+1) / 2 \beta}}
$$

and the integral $\mathscr{I}(\Delta t, \Delta F)$ is given by

$$
\mathscr{I}(\Delta t, \Delta F)=\int_{0}^{\infty} \frac{\nu^{(2 \alpha-\beta+1) / \beta}}{\mathscr{S}_{X}(\nu)} \exp \left(2 i \pi\left\{\nu \Delta t-\frac{\beta}{\beta+1} \nu^{(\beta+1) / \beta} \Delta F\right\}\right) d \nu
$$

Since we assumed that $M \in L^{1}\left(\mathbb{R}^{+}\right)$, the latter integral is absolutely convergent, and one obviously has $|\mathscr{I}(\Delta t, \Delta F)| \leq \mathscr{I}(0,0)$, so that

$$
\left|\mathscr{A}_{\left(t_{0}, F_{0}\right)}(t, F)\right| \leq \frac{1}{|\beta|}\left(F_{0} F\right)^{-(2 \alpha+1) / 2 \beta}\|M\|_{1}
$$

with equality if and only if $\Delta F=\Delta t=0$. Therefore, the ambiguity function may legitimately be used for detection purpose.

The integral $\mathscr{I}$ is again an oscillatory integral. In order to better understand its behavior, we use again a stationary phase argument. For the sake of simplicity, we set

$$
f(\nu)=2 \pi\left\{\nu \Delta t-\frac{\beta}{\beta+1} \nu^{(\beta+1) / \beta} \Delta F\right\} .
$$

When $\Delta t$ and $\Delta F$ are such that $f^{\prime}$ never vanishes, then an integration by parts argument may be used to yield upper bounds for the integral. Otherwise, let us 
denote by $\nu_{s}$ a stationary point ${ }^{\mathrm{e}}$ of $f$, i.e. $f^{\prime}\left(\nu_{s}\right)=0$. Assuming uniqueness of $\nu_{s}$, we have

$$
\mathscr{I}(\Delta t, \Delta F)=\mathscr{I}_{0}(\Delta t, \Delta F)+r(\Delta t, \Delta F)
$$

where

$$
\mathscr{I}_{0}(\Delta t, \Delta F)=\sqrt{2 \pi} e^{i \operatorname{sgn}\left(f^{\prime \prime}\left(\nu_{s}\right)\right) \pi / 4} \frac{M\left(\nu_{s}\right)}{\sqrt{\left|f^{\prime \prime}\left(\nu_{s}\right)\right|}} e^{i f\left(\nu_{s}\right)},
$$

the remainder $r(\Delta t, \Delta F)$ being bounded as in Lemma A.2 of Appendix A. The following result gives first indications on the existence of such stationary points, and the corresponding behavior of the ambiguity function.

Proposition 2.4. With the same notations as above, assume $M \in L^{1}\left(\mathbb{R}^{+}\right)$. Assume further that $\Delta F \neq 0$ and $\Delta t \neq 0$. Then,

(1) The integral in equation (2.19) admits a stationary point $\nu_{s}$ if and only if $\Delta t$ and $\Delta F$ have the same sign. $\nu_{s}$ is unique, and is given by

$$
\nu_{s}=\left(\frac{\Delta t}{\Delta F}\right)^{\beta} \text {. }
$$

(2) If $\Delta t$ and $\Delta F$ have the same sign, the leading term of the stationary phase approximation has modulus

$$
\left|\mathscr{I}_{0}(\Delta t, \Delta F)\right|=\sqrt{\frac{3}{8}}\left(\frac{\Delta F}{\Delta t}\right)^{\beta / 2} \frac{1}{\sqrt{|\Delta F|}} \frac{1}{\mathscr{S}_{X}\left(\left(\frac{|\Delta t|}{|\Delta F|}\right)^{\beta}\right)} .
$$

Proof. The first point results directly from the computation of the derivative of $f$, which vanishes if and only if $\Delta t=\nu^{1 / \beta} \Delta F$. For the second, we use the stationary phase lemma ${ }^{11}$ (see Lemma A.2 in the appendix).

Such an approximation turns out to be quite accurate, as we shall see in the numerical illustrations in Section 4.

\section{Continuous wavelet transform, and the line integral detection approach}

Wavelet transform has been introduced quite some time ago by A. Grossmann and J. Morlet ${ }^{10}$, in a context of analog signal analysis. Since then, many variations around wavelet expansions have been derived and used in different contexts. Nevertheless, we stick in the present work to a version close to the original one, which is best adapted to the considered problem because of its built-in translation and scaling invariance properties. Indeed, it may be seen easily ${ }^{20}$ that in the signal model (2.11) the $t_{0}$ parameter exactly plays the role of a time translation, and the $F_{0}$ parameter acts as a rescaling variable (more precisely, $F_{0}^{-1 /(\beta+1)}$ is a scale variable.)

${ }^{\mathrm{e}}$ notice that $\nu_{s}$ depends on $t, F, t_{0}, F_{0}$. 


\subsection{Continuous wavelet transforms}

Let us start by briefly recalling the main features of the version of continuous wavelet transform we shall be using in the sequel. More detailed analyses may be found in the literature. ${ }^{3,6,17}$ Let $\psi$ be an integrable and square-integrable function, such that $\hat{\psi}(\nu)=0$ for all $\nu \leq 0$, and that

$$
0<c_{\psi}:=\int_{0}^{\infty}|\hat{\psi}(\nu)|^{2} \frac{d \nu}{\nu}<\infty
$$

Functions satisfying the above properties are called progressive wavelets. Such wavelets may be used to provide alternative representations of signals as follows. Let $x$ be a square-integrable real-valued ${ }^{\mathrm{f}}$ function, and let $z_{x}$ denote its analytic signal (see Definition 2.2.) Then one may write

$$
z_{x}=\frac{1}{c_{\psi}} \int T_{x}(b, s) \psi_{(b, s)} \frac{d s d b}{s^{2}}
$$

where the functions $\psi_{(b, s)}$ are the wavelets, shifted and rescaled copies of $\psi$

$$
\psi_{(b, s)}(t)=\frac{1}{\sqrt{s}} \psi\left(\frac{t-b}{s}\right),
$$

and the coefficients $T_{x}(b, s)$ defined by

$$
T_{x}(b, s)=\left\langle x, \psi_{(b, s)}\right\rangle=\frac{1}{\sqrt{s}} \int_{-\infty}^{\infty} x(t) \bar{\psi}\left(\frac{t-b}{s}\right) d t
$$

form the Continuous wavelet transform $T_{x} \in L^{2}\left(\mathbb{R} \times \mathbb{R}^{+}, d b d s / s^{2}\right)$ of $x$. The integral in (3.27) runs over the half plane $(b, s) \in \mathbb{R} \times \mathbb{R}^{+}$. Obviously, $x$ may be recovered from its analytic signal as $x=\Re\left(z_{x}\right)$, where $\Re$ denotes the real part, so that Equation (3.27) provides a complete analysis-reconstruction scheme.

The wavelet transform may also be extended to the case of random signals. To see this, consider a second order continuous and stationary random process $X$ with spectral density $\mathscr{S}_{X}$, and assume $\mathscr{S}_{X} \in L^{\infty}$. Set $\tilde{\psi}_{s}(t)=\frac{1}{\sqrt{s}} \bar{\psi}\left(-\frac{t}{s}\right)$. Then it is easy to see ${ }^{15,16}$ that for any $s>0$, the process $T_{X}^{(s)}$ defined by the convolution

$$
T_{X}^{(s)}=\tilde{\psi}_{s} * X
$$

is itself second order continuous and stationary, and has spectral density $\nu \rightarrow$ $s|\hat{\psi}(s \nu)|^{2} \mathscr{S}_{X}(\nu)$. Hence, setting $T_{X}(b, s)=T_{X}^{(s)}(b)$, one may define the wavelet transform of the random signal $X$ as the family $T_{X}$ of random processes $\left\{T_{X}^{(s)}, s \in\right.$ $\left.\mathbb{R}^{+}\right\}$. If $\psi$ has zero integral, then $\mathbb{E}\left\{T_{X}(b, s)\right\}=0$ for all $b, s$. In addition, one easily sees ${ }^{3}$ that $\mathbb{E}\left\{T_{X}(b, s) \overline{T_{X}}\left(b^{\prime}, s^{\prime}\right)\right\}=\left\langle\psi_{\left(b^{\prime}, s^{\prime}\right)}, \mathscr{C}_{X} \psi_{(b, s)}\right\rangle$. Finally, if the noise is assumed gaussian as before, the wavelet transform is also gaussian.

In what follows, considering the situation where a signal $x$ has been embedded into a random noise $X$ as above (with fixed spectral density $\mathscr{S}_{X}$ and covariance

$\mathrm{f}_{\text {we shall always make this assumption from now on }}$ 
operator denoted by $\mathscr{C}_{X}$ ), we shall use a slightly different wavelet transform, the corresponding pre-whitened wavelet transform $\mathscr{W}_{x}$, defined as follows:

$$
\mathscr{W}_{x}(b, s)=\left\langle x, \mathscr{C}_{X}^{-1 / 2} \psi_{(b, s)}\right\rangle .
$$

Clearly enough, the pre-whitened wavelet transform of the random noise $X$ reduces to a classical wavelet transform of a white noise. In particular, using the same notations as above, one has

$$
\begin{aligned}
\mathbb{E}\left\{\mathscr{W}_{X}(b, s)\right\} & =0, \\
\mathbb{E}\left\{\mathscr{W}_{X}(b, s) \overline{\mathscr{W}_{X}}\left(b^{\prime}, s^{\prime}\right)\right\} & =\left\langle\psi_{\left(b^{\prime}, s^{\prime}\right)}, \psi_{(b, s)}\right\rangle, \\
\operatorname{Var}\left\{\mathscr{W}_{X}(b, s)\right\} & =\|\psi\|^{2} .
\end{aligned}
$$

The purpose of this transform is to obtain a constant variance wavelet transform.

\subsection{Wavelet transform of chirps}

We essentially follow here refs ${ }^{8,22}$. Let us consider a model signal of the form (2.5) where the amplitude and phase functions are assumed to be twice and four times continuously differentiable respectively, and assume that the amplitude $a(t)$ is slowly varying compared to the oscillations. Let us consider a complex-valued (progressive) analyzing wavelet, which we write in the form

$$
\psi(t)=a_{\psi}(t) e^{i \varphi_{\psi}(t)},
$$

and assume for the sake of simplicity that the (positive) amplitude $a_{\psi}$ is smooth and maximal at $t=0$, with $|\psi(0)|=1$, and that the wavelet is a linear phase one, i.e. is such that $\varphi_{\psi}^{\prime}(t)=\omega_{0}=2 \pi$. Then writing the continuous wavelet transform as an oscillatory integral, and approximating the integral by means of the stationary phase method (see Lemma A.2 in the appendix), we obtain the following approximate expression $^{8}$ for the wavelet transform of (2.5):

$$
T_{x}(b, s) \approx \sqrt{\frac{\pi}{2}} \frac{e^{i \pi \operatorname{sgn}\left(\Phi_{(b, s)}^{\prime \prime}\left(t_{s}\right)\right) / 4}}{\sqrt{s\left|\Phi_{(b, s)}^{\prime \prime}\left(t_{s}\right)\right|}} \bar{\psi}\left(\frac{t_{s}-b}{s}\right) z_{x}\left(t_{s}\right),
$$

where $\Phi_{(b, s)}(t)$ is the argument of the integrand, and the symbol $\operatorname{sgn}(t)$ stands for the sign of $t . t_{s}=t_{s}(b, s)$ is a stationary point of the integrand, i.e. a value of the argument such that $\Phi_{(b, s)}^{\prime}\left(t_{s}\right)=0$. In addition, it is assumed that for any $(b, s)$ under consideration, there exists one and only one such point, and that $\Phi_{(b, s)}^{\prime \prime}\left(t_{s}\right) \neq 0$ (otherwise, higher order approximations have to be used).

Such stationary points have a very simple intuitive meaning: they represent the values of the integrand for which the oscillations of the wavelet match perfectly those of the signal. The precision of the approximation in (3.35) depends on the "speed of variations" of the oscillations of the integrand compared to its amplitude. However, the integrand involves both the signal and the scaled wavelet. 
An alternative may be derived in the particular case of the so-called Morlet wavelet

$$
\psi(t)=e^{-t^{2} / 2 \sigma^{2}} e^{i \omega_{0} t},
$$

where the stationary phase argument may be refined ${ }^{g}$ to yield the following approximation

Proposition 3.1. Let $x$ be a chirp signal as in (2.5), and let $\psi$ be the Morlet wavelet. Then

$$
T_{x}(b, s)=\sqrt{\frac{\pi}{2}} \frac{e^{i \arctan \left(2 \sigma^{2} s^{2} \Phi_{(b, s)}^{\prime \prime}\left(t_{s}\right)\right) / 2}}{\sqrt{s \sqrt{\Phi_{(b, s)}^{\prime \prime}\left(t_{s}\right)^{2}+1 / \sigma^{4} s^{4}}}} \bar{\psi}\left(\frac{t_{s}-b}{s}\right) z_{x}\left(t_{s}\right)+r(b, s)
$$

where $r$ depends on derivatives of a (resp. $\varphi$ ) of order up to two (resp. four).

The proof of the proposition may be found in Appendix B, together with more precise details on the remainder. Notice that in the limit $\sigma \rightarrow \infty$, one recovers the previous expression (3.35). Notice also that the remainder is not uniform with respect to scale, which means that the precision of the approximation depends upon scale.

The approximate wavelet transforms in (3.35) and (3.37) are interesting in many respects. Let us simply point out that they naturally lead to the introduction of the following set of points

$$
\mathscr{R}=\left\{(b, s) \text { such that } t_{s}(b, s)=b\right\},
$$

termed the ridge of the wavelet transform. Indeed, if we forget for the sake of simplicity about the denominator in (3.35), we can see that $\left|T_{x}(b, s)\right|^{2}$ is locally maximum in the neighborhood of the ridge (this follows from the assumptions on the localization of the wavelet). In addition, it is readily verified that the ridge takes the form of a curve in the time-scale plane

$$
s=s_{r}(b)=\frac{\varphi_{\psi}^{\prime}(0)}{\varphi^{\prime}(b)},
$$

where the argument $\varphi$ (resp. $\varphi_{\psi}$ ) of the signal (resp. the wavelet) is defined in (2.5) (resp (3.34).) If the signal is locally monochromatic (see the discussion above), the function $s_{r}(b)$ is monotonic and we may represent the ridge using the reciprocal function $b=b_{r}(s)$.

An example of such localization around a ridge is given in Fig. 5, in the particular case of a power law chirp, as given in (2.5). In such a situation, the ridge takes the form of a curve in the $(b, s)$ half-plane, of equation

$$
b_{r}(s)=t_{0}-\left(\frac{2 \pi F_{0}}{\varphi_{\psi}^{\prime}(0)} s\right)^{-1 / \beta} \quad, \quad \text { or } \quad s_{r}(b)=F_{0}\left(t_{0}-b\right)^{\beta},
$$

with $s \in(0, \infty)$ and $b \in\left(-\infty, t_{0}\right)$.

g Taking advantage of the Gaussian amplitude. 


\subsection{Line integrals detection}

According to the previous expressions, the restriction of the wavelet transform to the ridge (also termed skeleton of the wavelet transform ${ }^{8}$ ) has a particular status, in the sense that its behavior is quite close to that of the analytic signal itself. ${ }^{8}$ To be more specific, notice that with our assumptions on the wavelet $\psi$ (namely, $\psi$ is the Morlet wavelet) we may write $\Phi_{(b, s)}^{\prime \prime}(t)=-\omega_{0} s_{r}^{\prime}(t) / s_{r}(t)^{2}$. Using the stationary phase approximation to $T_{x}$, it follows from equation (3.35) that

$$
T_{x}\left(b, s_{r}(b)\right) \approx \sqrt{\frac{\pi}{2 \omega_{0}}} \sqrt{\left|\frac{s_{r}(b)}{s_{r}^{\prime}(b)}\right|} e^{-i \frac{\pi}{4} \operatorname{sgn}\left(s_{r}^{\prime}(b)\right)} \overline{\psi(0)} z_{x}(b)
$$

(a similar expression may be obtained using the approximation obtained in Proposition 3.1). We now show how to take advantage of such expressions for constructing a corresponding detection algorithm

Remark 3.1. Notice also that according to the same approximations, we have

$$
\int\left|T_{x}\left(b_{r}(s), s\right)\right|^{2} \frac{d s}{s}=\int\left|\frac{s_{r}^{\prime}(b)}{s_{r}(b)}\right|\left|T_{x}\left(b_{r}(s), s\right)\right|^{2} d b=\frac{1}{2}\|x\|^{2}
$$

We are interested in detecting the presence of a frequency modulated signal from its wavelet transform. A now classical strategy ${ }^{3,9}$ consists in seeking the "optimal ridge", i.e. the ridge which concentrates optimally the time-scale energy, within a family of "candidate ridges." Parametrizing ridges as functions of the scale variable $s$, within a certain range $\left[s_{0}, s_{1}\right]$,

$$
s \in\left[s_{0}, s_{1}\right] \rightarrow \rho(s),
$$

one is led to seek the function $\rho$ which maximizes

$$
\Gamma[\rho]=\int_{s_{0}}^{s_{1}}\left|T_{x}(\rho(s), s)\right|^{2} \frac{d s}{s} .
$$

In the general situation, we may write

$$
\begin{aligned}
\Gamma[\rho] & \approx C \int_{s_{0}}^{s_{1}}\left|\psi\left(\frac{b_{r}(s)-\rho(s)}{s}\right)\right|^{2}\left|z_{x}\left(b_{r}(s)\right)\right|^{2}\left(\frac{s_{r}(\rho(s))}{s}\right)^{2} \frac{d s}{\left|s_{r}^{\prime}(\rho(s))\right|} \\
& \approx C \int_{b_{r}\left(s_{1}\right)}^{b_{r}\left(s_{0}\right)}\left|z_{x}(b)\right|^{2}\left|\psi\left(\frac{b-\lambda(b)}{s_{r}(b)}\right)\right|^{2}\left(\frac{s_{r}(\lambda(b))}{s_{r}(b)}\right)^{2}\left|\frac{s_{r}^{\prime}(b)}{s_{r}^{\prime}(\lambda(b))}\right| d b,
\end{aligned}
$$

where we have introduced the auxiliary function

$$
\lambda: b \in\left[b_{0}, b_{1}\right] \rightarrow \lambda(b)=\rho\left(s_{r}(b)\right) .
$$

Introducing the other auxiliary function $\nu_{r}: b \rightarrow \nu_{r}(b)=\frac{\omega_{0}}{s_{r}(b)}$ (which may be interpreted as instantaneous frequency), the latter expression reads

$$
\Psi[\rho] \approx C \int_{b_{r}\left(s_{1}\right)}^{b_{r}\left(s_{0}\right)}\left|z_{x}(b)\right|^{2}\left|\psi\left(\frac{\nu_{r}(b)}{\omega_{0}}(b-\lambda(b))\right)\right|^{2}\left|\frac{\nu_{r}^{\prime}(b)}{\nu_{r}^{\prime}(\lambda(b))}\right| d b .
$$


Therefore, if the considered wavelet $\psi$ is sufficiently localized around the origin $t=0$ so that

$$
\left|\psi\left(\frac{\nu_{r}(b)}{\omega_{0}}(b-\lambda(b))\right)\right|^{2}\left|\frac{\nu_{r}^{\prime}(b)}{\nu_{r}^{\prime}(\lambda(b))}\right| \leq|\psi(0)|^{2},
$$

for all $b \in\left[b_{0}, b_{1}\right]$, then

$$
\Gamma[\rho] \leq \Gamma\left[b_{r}\right],
$$

and the numerical optimization of $\Gamma$ will yield correct estimates for the seeked ridge. ${ }^{3}$

The situation we consider here is slightly different, in two respects. First, we have a parametric model at hand for the signal. Therefore, the space of potential ridges is a finite dimensional space (a two-dimensional space in the case of power law chirps). Second, the noise level is much higher than in the previously considered non-parametric situations. ${ }^{3}$

In the case of power law chirps, the ridge function takes the form

$$
s \rightarrow b_{t, F}(s)=t-\left(\frac{2 \pi F}{\omega_{0}} s\right)^{-1 / \beta}
$$

(recall that $\omega_{0}$ is a constant). Thus, we are led to consider "energy" functions of the form

$$
\Gamma(t, F)=\Gamma\left[b_{t, F}\right],
$$

and seek maxima of such a two variables function with respect to $t$ and $F$.

Remark 3.2. According to the stationary phase approximation (3.35), given a candidate ridge $\rho$, we may write

$$
\left|T_{x}(\rho(s), s)\right| \approx C_{F_{0}}(s)\left|\psi\left(\left(\frac{\omega_{0}}{s^{\beta+1}}\right)^{1 / \beta} \Delta F-\frac{\Delta t}{s}\right)\right| .
$$

Therefore, if we still assume that $|\psi|$ is maximum at the origin, we see that this value may be attained only if $\Delta t$ and $\Delta F$ have the same sign. Under such a condition, one easily sees that the candidate ridge and the true ridge do intersect, which results in significantly large values for the line integral. This is reminiscent of the result of Proposition 3.1, and we shall see later on in the numerical simulations that the $\Gamma$ functions shows a behavior similar to (a somewhat blurred version of) the ambiguity function $\mathscr{A}$.

The statistical behavior of the line integrals is more difficult to understand. Under the $H_{0}$ hypothesis (noise only), the prewhitened wavelet transform of the signal $y$ analogous to an ordinary wavelet transform of a gaussian white noise. Hence, all coefficients $\mathscr{W}_{y}(b, s)=\mathscr{W}_{X}(b, s)$ are also gaussian, and their square modulus $\left|\mathscr{W}_{y}(b, s)\right|^{2}$ follow a centered $\chi^{2}$ distribution. The line integral is therefore a sum of $\chi^{2}$ variables, but the latter being highly correlated, the distribution of the line 
integrals is very difficult to characterize precisely. The same holds true under the $H_{1}$ hypothesis: $\left|\mathscr{W}_{y}(b, s)\right|^{2}=\left|A \mathscr{W}_{x_{\theta}}(b, s)+\mathscr{W}_{X}(b, s)\right|^{2}$ is distributed according to an uncentered $\chi^{2}$ distribution (i.e. as a sum of squares of uncentered Gaussians), and the distribution of line integrals appears to be quite difficult to characterize (sum of correlated centered or non-centered $\chi^{2}$ variables). Further work is clearly needed at this point, maybe following the lines developed by Hory and collaborators. ${ }^{13}$

\section{Target application: detection of gravity waves}

As target application, we consider the problem of detection/estimation of gravitational waves at interferometric detectors. The existence of such waves was predicted long ago $^{23}$ by general relativity, but no direct observation could be done so far. High scale experiments are currently being prepared, which should be able to provide such direct observations. Among the potential sources for gravity waves, the waves generated by coalescing binary star systems are particularly appealing, since the corresponding waveform is fairly well-known, and may be given (to some approximation degree) an explicit expression: in the so-called restricted post-Newtonian approximation, a binary system of masses $m_{1}$ and $m_{2}$ (we denote by $M$ the total mass and by $\mu$ the reduced mass), coalescing at time $t_{0}$ generates a chirp of the form

$$
x(t)=a(t) \cos g(t)
$$

where the amplitude function is of the form

$$
a(t)=A\left(t_{0}-t\right)^{-1 / 4}
$$

and the phase function reads

$$
\begin{aligned}
g(t)= & \phi_{c}-\frac{2}{\eta}\left[\left(\eta M\left(t_{0}-t\right)\right)^{5 / 8}+\left(\frac{3517}{8064}+\frac{55}{96} \eta\right)\left(\eta M\left(t_{0}-t\right)\right)^{3 / 8}\right. \\
& \left.-\frac{3 \pi}{4}\left(\eta M\left(t_{0}-t\right)\right)^{1 / 4}+\left(\frac{9275495}{14450688}+\frac{284875}{258048} \eta+\frac{1855}{2048} \eta^{2}\right)\left(\eta M\left(t_{0}-t\right)\right)^{1 / 8}\right],
\end{aligned}
$$

with $\eta=\frac{\mu}{M}$, and $\phi_{c}$ a constant representing the phase of the waveform at coalescence time. The amplitude constant $A$ depends on the masses $M$ and $\mu$, and is also inversely proportional to the distance at which the event took place. An example of such a signal is displayed in Figure 1.

Such expressions result from asymptotic approximations ${ }^{\mathrm{h}}$, and it is sometimes convenient to content oneself with the first order (i.e. Newtonian) approximation, which features the same amplitude function, and a phase function of the form

$$
g(t)=\phi_{c}-\frac{2}{\eta}\left[\left(\eta M\left(t_{0}-t\right)\right)^{5 / 8}\right]
$$

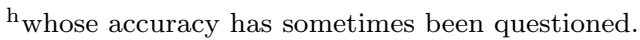




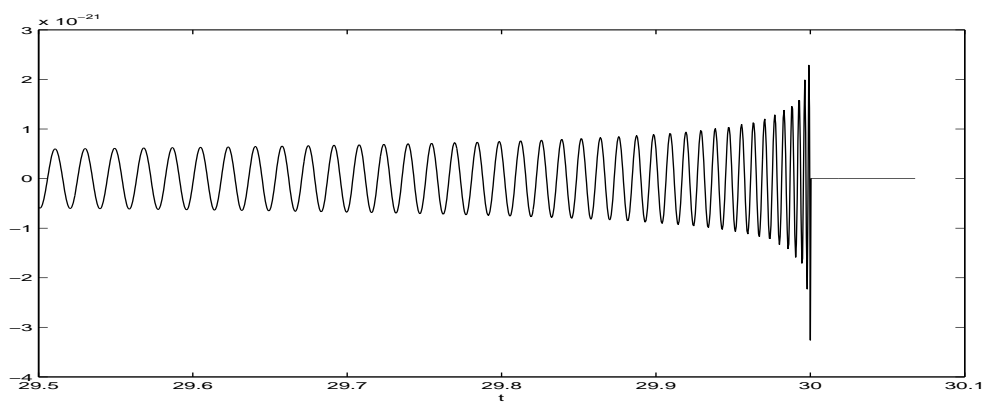

Fig. 1. An example of power law chirp signal generated by the coalescence of a binary system.

which only depends on the parameter

$$
\mathscr{M}=\left(M^{2} \mu^{3}\right)^{1 / 5},
$$

called the chirp mass, rather than on $M$ and $\mu$ independently. Such a model falls in the class of the power law chirps we described above.

The considered detection problem is made significantly difficult because of the presence of noise, with a very low signal to noise ratio. The noise is generally considered Gaussian, zero mean and stationary, and the power spectral density is assumed to be known. We shall work with a spectral density (corresponding to the VIRGO $^{2}$ experiment) of the form

$$
\mathscr{S}_{X}(\nu)=S_{1} \nu^{-5}+S_{2} \nu^{-1}+S_{3}\left(1+\left(\frac{\nu}{500}\right)^{2}\right)+S_{v}(\nu)
$$

The first terms represent pendulum thermal noise, mirror thermal noise and shot noise respectively (the seismic noise has been neglected). The term $S_{v}$ represents the "violin modes" of the thermal noise (i.e. peaks in the spectrum), namely the resonances of the various pieces of the detector, excited by thermal noise, and is given by

$$
S_{v}(\nu)=\sum_{n=1}^{\infty} \frac{1}{\nu} \frac{K}{\left(\nu^{2}-\nu_{n}^{2}\right)^{2}+\phi^{2} \nu_{n}^{4}},
$$

where $K$ and $\phi$ are global constants. The numerical values of the constants depend on physical parameters of the experiment. The simulations presented here have always been done with the same set of values, and violin modes have been suppressed. The spectral density is shown in Fig. 2.

Remark 4.1. Because of the particular shape of the spectral density (which diverges as $\nu \rightarrow 0$ and $\nu \rightarrow \infty$ ), the contribution of very low and very high frequencies is negligible (which is fortunate, the physical meaning of such divergencies being highly questionable). Therefore, it is legitimate to introduce infrared and ultraviolet 


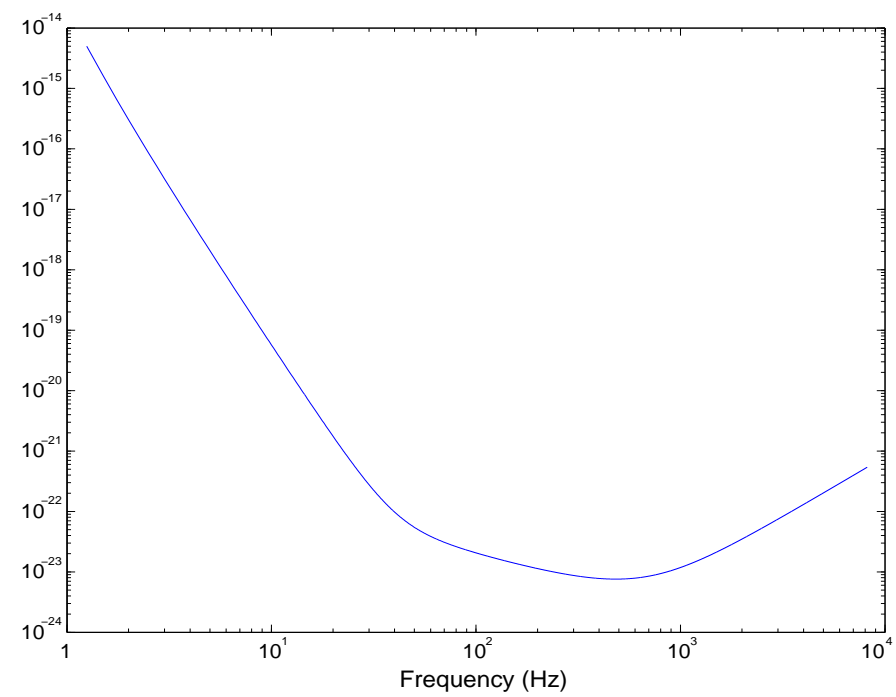

Fig. 2. Model for the noise spectral density, taken from the VIRGO experiment (the "violin modes" have been neglected.)

cutoffs in the numerical simulations. Also, the discussion regarding the integrability of the function $M$ becomes purely academic in such a context.

We applied the two detection strategies to the considered class of signals, for various values of the parameters (star masses, distance,...). Gravitational signals corresponding to Newtonian approximation (power law chirps) were generated, with sampling frequency $\eta=2000 \mathrm{~Hz}$, and embedded into gaussian stationary coloured noise with the prescribed spectral density (4.51) (for the sake of simplicity, the violin modes have not been considered), with various values of the signal to noise ratio (which corresponds to gravitational collapses taking place at various distances from the detector). As mentioned in Remark 4.1, infrared and ultraviolet cutoffs have been introduced. The results are presented below.

\subsection{The cross-ambiguity function}

We first consider the ambiguity function as defined in Definition 2.1, adapted to the considered signal class.

Let us remark that thanks to the divergent behavior of the spectral density $\mathscr{S}_{X}$ at the origin and at infinity, the function $M$ introduced in (2.16) is integrable. Indeed, we have

$$
\|M\|_{1}=\int_{0}^{+\infty} \frac{\nu^{\frac{2 \alpha-\beta+1}{\beta}}}{\mathscr{S}_{X}(\nu)} d \nu=\int_{0}^{+\infty} \frac{\nu^{-\frac{7}{3}}}{S_{1} \nu^{-5}+S_{2} \nu^{-1}+S_{3}\left(1+\left(\frac{\nu}{500}\right)^{2}\right)} d \nu<\infty .
$$

Therefore, the discussion of Section 2.3 applies. The considered numerical values 

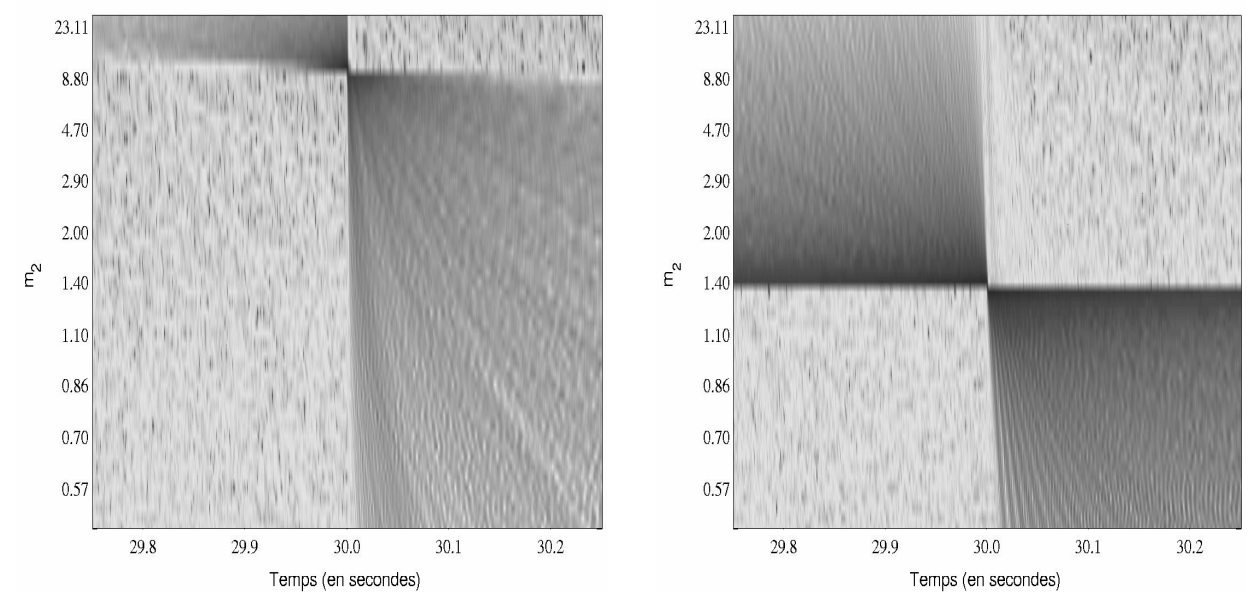

Fig. 3. Two examples of generalized cross-ambiguity function, for the case of power-law chirps corresponding to gravity waves in the Newtonian approximation: $m_{1}=m_{2}=10 M_{\odot}$ (left) and $m_{1}=m_{2}=1.4 M_{\odot}$ (right).

of $\alpha$ and $\beta$ yield the following approximate behavior for the peak value of the ambiguity function:

$$
\left|\mathscr{A}_{\left(t_{0}, F_{0}\right)}(t, F)\right| \leq \frac{8}{3} F_{0}^{-4 / 3} \int_{0}^{\infty} \frac{\nu^{-7 / 3}}{\mathscr{S}_{X}(\nu)} d \nu=C F_{0}^{-4 / 3} .
$$

Numerical simulations (not presented here) confirm such predictions. This provides a simple way to normalize the ambiguity function

Furthermore, Proposition 2.4 leads us to expect significantly different behaviors for the ambiguity function, depending on the relative sign of $\Delta t$ and $\Delta F$. Such a behavior may be seen explicitely in the two illustrations of Figure 3, which represent the $\mathscr{A}$ function (displayed in gray levels) versus the time parameter $t$ and the mass parameter $m_{2}$ (or the frequency parameter $F$ ), in two situations: $m_{1}=m_{2}=$ $10 M_{\odot}$ (left) and $m_{1}=m_{2}=1.4 M_{\odot}$ (right). In both cases, the parameter space is clearly divided into four quadrants, the boundaries of which correspond essentially to the correct values of parameters. It may be seen that the left bottom and right top quadrants correspond to situations where $\Delta t$ and $\Delta F$ have equal sign, which manifests itself by higher values of the ambiguity function. Conversely, the left top and right bottom quadrants correspond to situations where $\Delta t / \Delta F<0$, and the absence of stationary point in the argument of the integrand of $\mathscr{A}$ yields significantly smaller values.

The same may be seen on the slices of the ambiguity function $\left(m_{1}=m_{2}=\right.$ $10 M_{\odot}$ ) displayed in Figure 4 . Both slices display a significantly assymetric behavior; the decay appears to be much faster when $\Delta t$ and $\Delta F$ do not have equal signs.

The ambiguity functions exhibit a sharp peak precisely at the point $t=$ $t_{0}, F=F_{0}$. This remark is the main point for the ambiguity function-based de- 

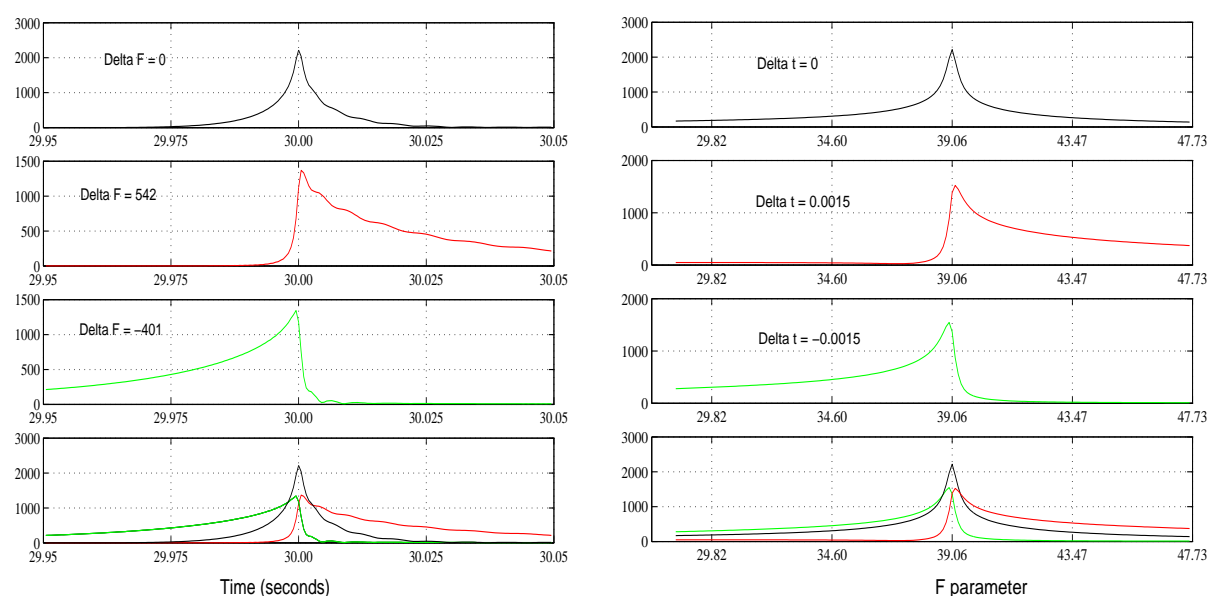

Fig. 4. Slices of the ambiguity function $\mathscr{A}$ corresponding to the situation $m_{1}=m_{2}=10 M_{\odot}$. Left: fixed $F$ slice for three different values of $F: \Delta F \approx 0$ (top), $\Delta F>0$ (middle top) and $\Delta F<0$ (middle bottom); the fourth plot is a superimposition of the other three. Right: fixed $t$ slice for three different values of $t: \Delta t \approx 0$ (top), $\Delta t>0$ (middle top) and $\Delta t<0$ (middle bottom).

tection/estimation algorithm.

Remark 4.2. Additional numerical simulations (still in the absence of noise, not displayed here) have shown that the precision in the determination of the parameters $t_{0}$ and $F_{0}$ is quite good (relative errors less than one percent). Clearly, such a precision depends highly on the sampling of the parameter space. In our numerical experiments, we have used a sampling rate equal to the signal's sampling rate for the $t$ parameter, and a (non-uniform) sampling of the $F$ parameter. All together, it seems that approximately one thousand of different values of $F$ are necessary to reach a correct precision.

Remark 4.3. At this point, it is worth coming back to the different models for gravity waves. We have essentially used here the Newtonian model, in which the masses parameters only appear through $F$. More realistic (post Newtonian) models take masses into account, and the $\left(m_{1}, m_{2}\right)$ space has to be sampled, rather than the $F$ space. This results in a much larger number of parameters to examine, and therefore to a much more complex algorithm. Hierarchical methods have been proposed to circumvent this problem: a first rough estimate is followed by a more refined one, in the neighborhood of the parameters estimated by the first step. A possible idea could also be that the first step could be performed using wavelet line integrals. ${ }^{19}$ This method is discussed below. 

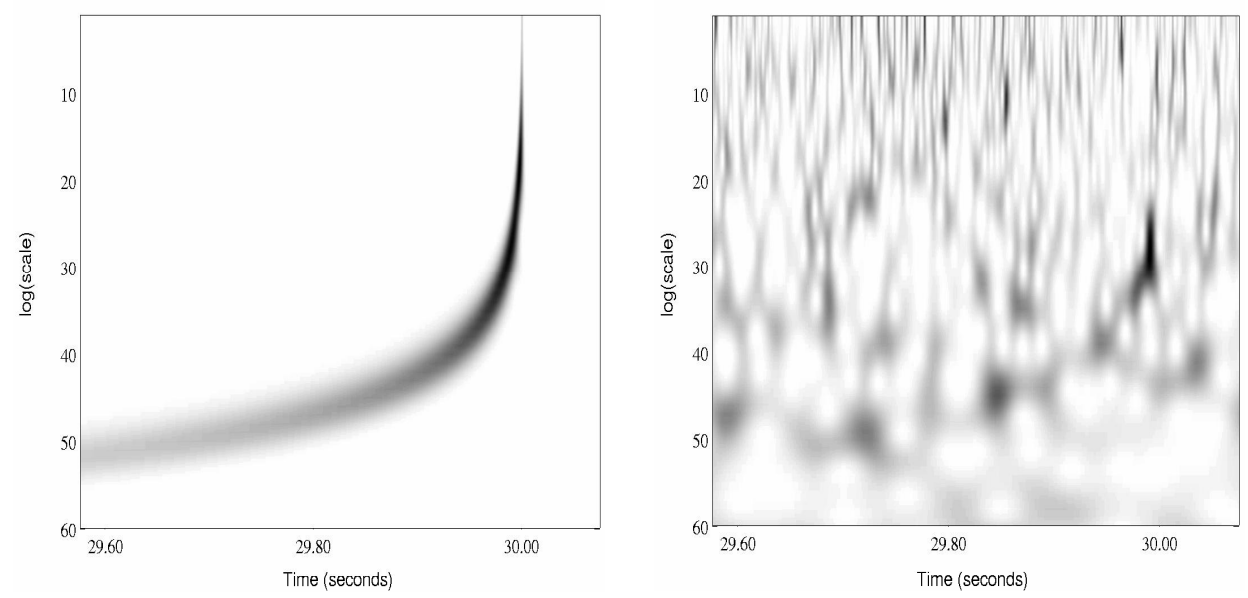

Fig. 5. Pre-whitened wavelet transforms of pure (left) and noisy (right) power law chirps. $m_{1}=$ $m_{2}=10 M_{\odot}$. The horizontal variable is the time variable, and the vertical one is the scale variable. Square modulus of the wavelet transform is encoded with gray levels.

\subsection{Wavelet transform line integrals}

We now consider the approach based upon ridge detection from pre-whitened wavelet transform. As stressed in Remark 4.3, the wavelet transform line integral method may be considered as a possible first step for detecting the presence of signal, even in situations where the signal is not completely matched to the model.

Remark 4.4. We shall use here the localization properties of the pre-whitened wavelet transform of power law chirp signals near corresponding ridges. Such localization properties have only been established in the case of the ordinary wavelet transform. However, the prewhitened wavelet transform $\mathscr{W}_{x}$ of a signal $x$ is nothing but the ordinary wavelet transform of $\mathscr{C}_{X}^{-1 / 2} x$. Therefore, it may be seen that if the function $1 / \mathscr{S}_{X}$ is smooth enough, in the domain of the time-scale half-plane in which the ridge approximations are correct, the property of localization near the ridge is preserved.

The localization properties of the (pre-whitened) wavelet transform of power law chirps is illustrated in Fig. 5, where the prewhitened transforms of a pure power-law chirp and a noisy one are displayed. The localization properties appear clearly in the pure case (left image), while the presence of noise seems to corrupt completely the transform of the signal in the noisy case.

However, detection is still possible in such situations, as illustrated by Fig. 6: the line integral function $\Gamma$ (represented with gray levels, as a function of the $t$ and $F$ variables) exhibits a well defined maximum in the neighborhood of the correct values $t=t_{0}, F=F_{0}$.

As stressed before, the objective of the line integral function $\Gamma$ is to provide a 


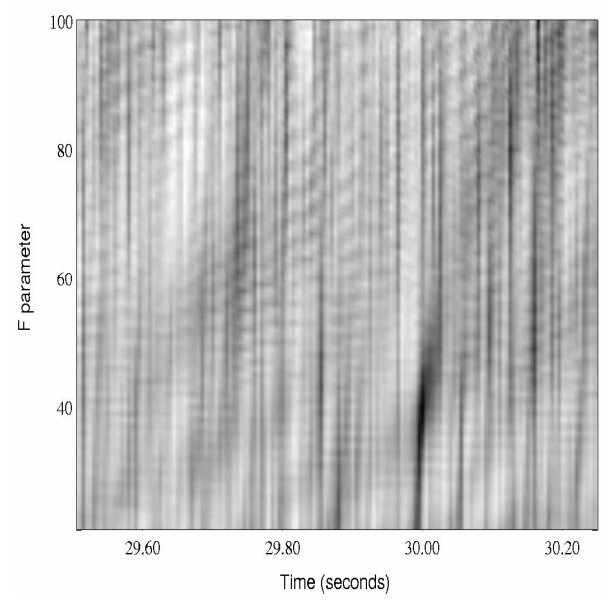

Fig. 6. Line integral for the noisy wavelet transform displayed in Figure 5 (right image).

robust alternative to the ambiguity function $\mathscr{A}$, at the price of a performance loss. Such a behavior appears clearly in Figures 7 and 8 (to be compared with Figures 3 and 4 , which correspond to the same parameter values; notice however that in 8 , the values of $\Delta F$ in the left hand figure have been taken much larger than in Figure 4; with the same $\Delta F$ as in 4 , the curves would have been undistinguishable): the variations of the line integral function are much slower than those of the ambiguity function. More precisely, variations with respect to the time parameter $t$ are comparable, while the variations with respect to the frequency parameter $F$ are much slower. This is precisely one of the goals of this approach. Slower variations imply that it is not necessary any more to sample densely the $\Gamma$ function (as was the case for the $\mathscr{A}$ function), which results in smaller computational burden. As a matter of comparison with the ambiguity function approach, let us point out that in this case, the number of values of $F$ to consider in order to get an accurate estimate of the true value was less than 100, instead of a thousand or so in the ambiguity function approach.

\subsection{Detection/estimation}

The numerical results on generalized cross-ambiguity functions and wavelet transform line integrals in the absence of noise, presented in the Sections 4.2 and 4.1, show the main features of the two approaches. The generalized ambiguity functions essentially feature much faster variations, and therefore a very significant peak at values of the parameters coinciding with the values of the signal. On the other hand, the wavelet transform line integral varies more slowly, and therefore may be expected to provide more robust estimates for the parameters, at least within a certain range of values of the signal to noise ratio.

In order to test the performances of the detector, simulations were run for 


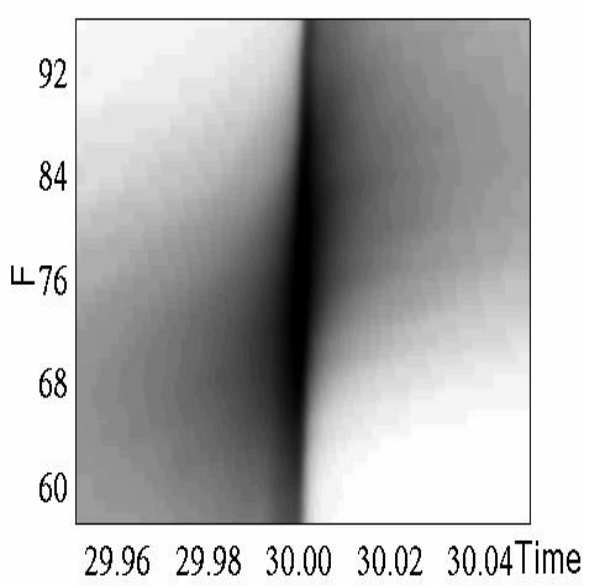

Fig. 7. Gray levels representation of the ridge detector, in the neighborhood of the correct values $\left(t_{0}, F_{0}\right)=(30,76.10)\left[m_{1}^{0}=10 M_{\odot}, m_{2}^{0}=1.4 M_{\odot}\right]$
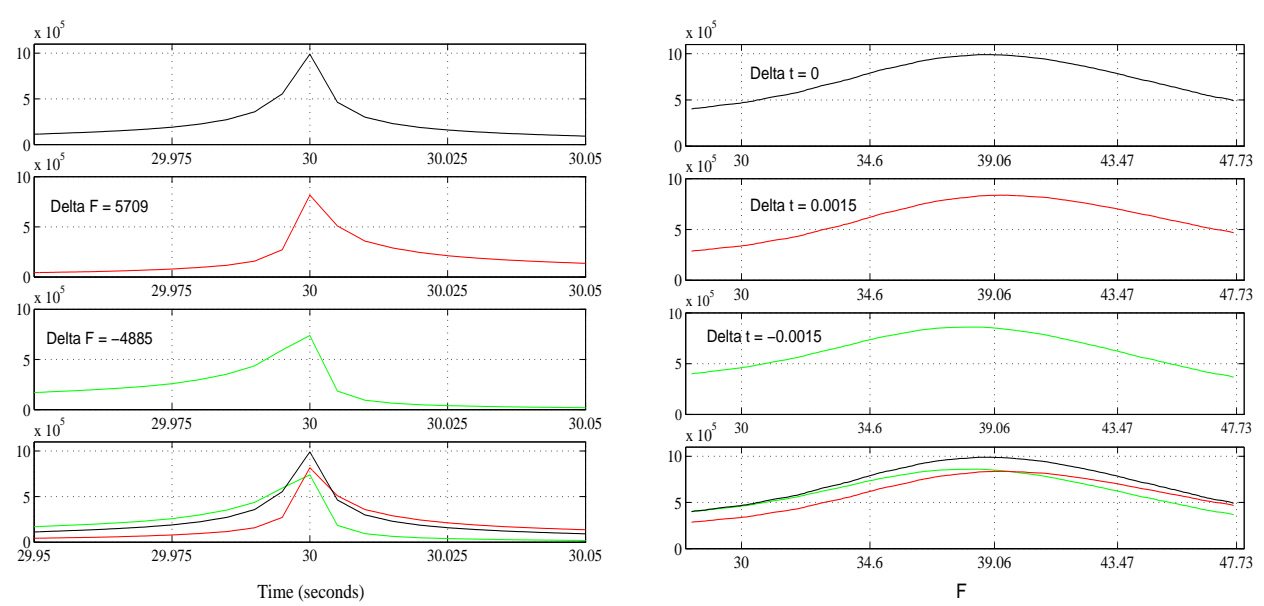

Fig. 8. Slices from the ridge detector $\Gamma(t, F)$. Left: fixed $F$ slices in three situations : $\Delta F=0$ (top), $\Delta F>0$ (middle), $\Delta F<0$ (bottom).

Right: fixed $t$ slices in three situations : $\Delta t=0$ (top), $\Delta t>0$ (middle), $\Delta t<0$ (bottom); the fourth figure is a superimposition of the other three.

various values of the masses and distance parameters (hence various values of the SNR), and the two approaches were used. For short, we will denote by $\mathscr{A}$ detector the detector based on ambiguity functions, and by $\mathscr{R}$ detector the detector based upon wavelet transform ridges. We present here the following comparisons. For a given configuration (masses and distance), the $\mathscr{A}$ detector was used first, with several values for the detection threshold, and false alarm and missed detections 
were recorded for all values of the threshold. For the sake of comparison, two versions for the $\mathscr{A}$ detectors were considered: a finely sampled one $\mathscr{A}_{f}$ (881 different values of $F$ ) and a coarser one $\mathscr{A}_{c}$ (160 different values of $F$.)

Then the $\mathscr{R}$ detector was used, with values of the threshold corresponding to the same false alarm rates as with the ambiguity function detector. The number of different values of $F$ was set to 160 (i.e. the same number as the coarsely sampled detector $\mathscr{A}_{c}$.)

Three configurations were tested: $m_{1}=m_{2}=1.4 M_{\odot}$ (corresponding to $F_{0} \approx$ 133.47), for three values of the distance $R, m_{1}=1.4 M_{\odot}, m_{2}=10 M_{\odot}$ (i.e. $F_{0} \approx$ 76.10 ), for two different values of $R$, and $m_{1}=m_{2}=10 M_{\odot}$ (i.e. $F_{0} \approx 39.06$ ), for three different values of $R$ ). In each situation, three different values of thresholds were considered, corresponding to 1, 2 and 3 false alarms per second respectively. The number of detections and missed detections was recorded in all situations.

The results obtained in these configurations are displayed in Table 1. As expected, the best results are generally obtained using the finely sampled detector $\mathscr{A}_{f}$. Nevertheless, in the third situation, no significant difference between $\mathscr{A}_{c}$ and $\mathscr{A}_{f}$ may be seen: in this range of values of $F_{0}$, the sampling of $F$ could presumably be made even coarser without much damage.

The comparison between the coarsely sampled $\mathscr{A}$ detector and the $\mathscr{R}$ detector does not seem to lead to definite conclusions, in the sense that none of the two approaches may be considered better than the other in all situations. For small values of the masses (i.e. high frequency signals), the ridge detector $\mathscr{R}$ seems to outperform the coarsely sampled ambiguity function detector $\mathscr{A}_{c}$. However, for larger values of the mass parameters, this behavior tends to disappear. Since we lack a precise statistical decision theory for ridge detectors, it is difficult to provide simple explanations for such a behavior. Nevertheless, let us mention that the approximations we have made concerning the oscillatory integrals defining the wavelet transform seems to be fairly precise in the frequency region around $100 \mathrm{~Hz}$; this could be a (partial) explanation for the appropriateness of the $\mathscr{R}$ detection scheme in that region.

\section{Conclusions}

We have studied in this paper two different approaches for the detection/estimation of power law chirps embedded into stationary random noise: the classical matched filter based approach, and an alternative based upon wavelet transform line integrals. ${ }^{12}$ We have shown that the latter may be seen as a "blurred" version of the former, in the sense that it provides a suboptimal alternative, which turns out to be more robust and probably more efficient from a computational point of view. An additional difficulty is introduced when it comes to the development of a corresponding decision theory. While the detection theory corresponding to ambiguity function (in other words, matched filters banks) is fairly well understood, some extra work is required to develop a corresponding theory for line integrals 


\begin{tabular}{|c|c|c|c|c|c|}
\hline $\begin{array}{l}\text { Masses } \\
\left(\text { in } M_{\odot}\right)\end{array}$ & Distance & $\begin{array}{l}\text { False alarms } \\
\text { (per second) }\end{array}$ & $\begin{array}{l}\text { Detections } \\
\mathscr{R} \text { detector }\end{array}$ & $\begin{array}{l}\text { Detections } \\
\mathscr{A}_{c} \text { detector }\end{array}$ & $\begin{array}{l}\text { Detections } \\
\mathscr{A}_{f} \text { detector }\end{array}$ \\
\hline \multirow{9}{*}{$\begin{array}{l}m_{1}=1.4 \\
m_{2}=1.4\end{array}$} & & 1 & 100 & 55 & 100 \\
\hline & $R=10 \mathrm{Mpc}$ & 2 & 100 & 65 & 100 \\
\hline & $\mathrm{SNR}=13.1$ & 3 & 100 & 70 & 100 \\
\hline & & 1 & 57 & 25 & 100 \\
\hline & $R=15 \mathrm{Mpc}$ & 2 & 67 & 30 & 100 \\
\hline & $\mathrm{SNR}=8.74$ & 3 & 79 & 45 & 100 \\
\hline & & 1 & 12 & 15 & 96 \\
\hline & $R=20 \mathrm{Mpc}$ & 2 & 22 & 18 & 97 \\
\hline & $\mathrm{SNR}=6.55$ & 3 & 34 & 23 & 98 \\
\hline \multirow{6}{*}{$\begin{array}{l}m_{1}=10 \\
m_{2}=1.4\end{array}$} & & 1 & 70 & 95 & 100 \\
\hline & $R=45 \mathrm{Mpc}$ & 2 & 76 & 97 & 100 \\
\hline & $\mathrm{SNR}=6.77$ & 3 & 81 & 99 & 100 \\
\hline & & 1 & 24 & 55 & 96 \\
\hline & $R=60 \mathrm{Mpc}$ & 2 & 28 & 60 & 97 \\
\hline & $\mathrm{SNR}=5.08$ & 3 & 34 & 66 & 98 \\
\hline \multirow{9}{*}{$\begin{array}{l}m_{1}=10 \\
m_{2}=10\end{array}$} & & 1 & 83 & 99 & 99 \\
\hline & $R=145 \mathrm{Mpc}$ & 2 & 86 & 99 & 99 \\
\hline & $\mathrm{SNR}=5.18$ & 3 & 87 & 99 & 99 \\
\hline & & 1 & 65 & 97 & 98 \\
\hline & $R=150 \mathrm{Mpc}$ & 2 & 73 & 99 & 99 \\
\hline & $\mathrm{SNR}=5.00$ & 3 & 77 & 99 & 99 \\
\hline & & 1 & 60 & 97 & 97 \\
\hline & $R=160 \mathrm{Mpc}$ & 2 & 67 & 98 & 98 \\
\hline & $\mathrm{SNR}=4.69$ & 3 & 69 & 99 & 99 \\
\hline
\end{tabular}

Table 1. Results of simulations using the three detection schemes: coarse and fine ambiguity function detectors $\mathscr{A}_{c}$ and $\mathscr{A}_{f}$, and wavelet ridge detection $\mathscr{R}$. The indicated value of SNR corresponds to the optimal signal to noise ratio for ambiguity function detection scheme. The last three columns give the number of detections (out of 100 possible), when the threshold is set so as to yield a fixed false alarm rate (given in the third column.)

(recall that the latter are constructed as sums of very correlated uncentered $\chi^{2}$ variables). This point is an important extension of the current work, which we plan to investigate in the future. Another possible extension of this work concerns the use of reassigned wavelet transforms $s^{7,9,14}$ instead of the wavelet transform, for optimizing the time-frequency localization, as proposed by Chassande-Mottin and Flandrin. ${ }^{5}$

From the point of view of the gravity waves detection, our results seem to indicate that wavelet transform line integrals could be considered an interesting alternative approach for online analysis of experimental signal. It appears to compare 
well with sparsely sampled matched filter banks (ambiguity functions), at least in some domains of values of the parameters. The suboptimality of the approach comes together with an increased robustness, which allows subsampling of the parameter space. In addition, such approaches should be much less sensitive to possible departures of the true signals from the restricted post Newtonian approximations which are used by cosmologists. This is another point which we plan to study in the future. However, let us also point out that the simulations we have presented in this paper are based on a noise model with smooth spectral density (the assumption is necessary in order to use stationary phase approximations on the prewhitened wavelet transform). In particular, the "violin modes" of the detector noise (i.e. periodic components) have not been taken into account. Incorporating such violin modes could result in a lowering of the performances of the algorithm.

\section{Acknowledgment}

We wish to thank E. Chassande-Mottin and J.M. Innocent for stimulating discussions. M. Morvidone is supported by a grant of the Fondo para el Mejoramiento de la Calidad Universitaria (FOMEC) de la República Argentina (Proyecto FOMEC 323). This work was partly supported by the "Research and Training Network" Harmonic Analysis and Statistics for Signal and Image Processing (HASSIP) of the European Commission. Part of this work was done when M. Morvidone was visiting the Signal Processing group of Napier University of Edimburgh, in the framework of the "Training and Research on Advanced Computing Systems" (TRACS) programme at EPCC (grant number HPRI-CT-1999-00026.)

\section{Appendix A. Proofs or Propositions 2.2 and 2.3, and Corollary 2.1}

Let us start with the proof of Proposition 2.2. Consider a signal of the form $a(t) \cos (\varphi(t))$, and assume that the assumptions of Proposition 2.2 hold true. Then it suffices to show that the Fourier transform of the function $t \rightarrow a(t) e^{-i \varphi(t)}$ is bounded as in the proposition. To show this, let $\nu \in \mathbb{R}^{+}$, and consider the integral

$$
\mathscr{I}=\int_{t_{1}}^{t_{2}} x(t) e^{-2 i \pi \nu t} d t
$$

where the interval $\left[t_{1}, t_{2}\right]$ is the support of $a$. By integrating by parts, and setting $\tilde{a}(s)=i a(s) /\left(\varphi^{\prime}(s)+2 \pi \nu\right)$ (which is legitimate since $\varphi^{\prime}$ is bounded from below and $\nu>0$ ), we obtain (the first term cancels because of the support properties of $a$ )

$$
|\mathscr{I}|=\left|\int_{t_{1}}^{t_{2}} \tilde{a}^{\prime}(t) e^{-i(\varphi(t)+2 \pi \nu t)} d t\right| \leq \int_{t_{1}}^{t_{2}}\left|\frac{a^{\prime}(t)}{\left(\varphi^{\prime}(t)+2 \pi \nu\right)}-\frac{a(t) \varphi^{\prime \prime}(t)}{\left(\varphi^{\prime}(t)+2 \pi \nu\right)^{2}}\right| d t
$$

Therefore, we obtain

$$
|\mathscr{I}| \leq \int_{t_{1}}^{t_{2}}\left|\frac{a^{\prime}(t)}{\varphi^{\prime}(t)}\right| d t+\int_{t_{1}}^{t_{2}}\left|\frac{a(t) \varphi^{\prime \prime}(t)}{\varphi^{\prime}(t)^{2}}\right| d t \leq\|a\|_{1}\left(\frac{\left\|a^{\prime}\right\|_{1}}{\|a\|_{1}}\left\|\frac{1}{\varphi^{\prime}}\right\|_{\infty}+\left\|\frac{\varphi^{\prime \prime}}{\left(\varphi^{\prime}\right)^{2}}\right\|_{\infty}\right),
$$


which yields the result.

We shall also make use of the following results.

Lemma A.1 Let $\beta \in \mathbb{C}, \Im(\beta)>0$, and let $A \in C_{0}^{2}$. Then

$$
\int_{-\infty}^{\infty} A(t) e^{i \beta t^{2}} d t=\sqrt{\frac{\pi}{|\beta|}} e^{-i \arg (\beta) / 2} A(0)+R(\beta, A),
$$

where

$$
|R(\beta, A)| \leq \frac{5}{4}\left\|A^{\prime \prime}\right\|_{\infty} .
$$

The lemma is easily proved using a change of variables. ${ }^{11}$ The second result is known as the stationary phase lemma, and results from Lemma A.1 above.

Lemma A.2 Let $M \in C_{0}^{2}$ and $f \in C^{5}$, and let $t_{s}$ be such that $f^{\prime}\left(t_{s}\right)=0$ and $f^{\prime \prime}\left(t_{s}\right) \neq 0$. Then

$$
\int_{-\infty}^{\infty} M(t) e^{i f(t)} d t=\sqrt{2 \pi} \frac{M\left(t_{s}\right)}{\sqrt{\left|f^{\prime \prime}\left(t_{s}\right)\right|}} e^{i \pi \operatorname{sgn}\left(f^{\prime \prime}\left(t_{s}\right)\right) / 4}+r,
$$

where

$$
|r| \leq \frac{5 \sqrt{\pi}}{4}\left\|B\left[f-f\left(t_{s}\right), M\right]\right\|_{\infty},
$$

the function $B$ being defined as follows: for $a \in C^{2}$ and $g \in C^{5}$

$$
\begin{aligned}
B[g, a] & =\left(\frac{|g|^{1 / 2}}{g^{\prime}}\right)^{3}\left\{a^{\prime \prime}-3 a^{\prime} \frac{g^{\prime \prime} g-\frac{1}{2}\left(g^{\prime}\right)^{2}}{|g|^{3 / 2}} \frac{\sqrt{|g|}}{g^{\prime}}\right. \\
& \left.+a\left[3\left(\frac{g\left(g^{\prime \prime}\right)^{2}-\frac{1}{2}\left(g^{\prime}\right)^{2}}{|g|^{3 / 2}} \frac{\sqrt{|g|}}{g^{\prime}}\right)^{2}-\frac{g^{\prime \prime \prime} g^{2}-\frac{3}{2} g^{\prime} g^{\prime \prime} g+\frac{3}{4}\left(g^{\prime}\right)^{3}}{|g|^{5 / 2}} \frac{\sqrt{|g|}}{g^{\prime}}\right]\right\}
\end{aligned}
$$

Again, the proof follows from a suitable change of variable (namely, $u(t)=$ $\left.\operatorname{sgn}(t) \sqrt{\left(f(t)-f\left(t_{s}\right)\right) / f^{\prime \prime}\left(t_{s}\right)}\right)$ and the use of Lemma A.1. The estimation of the remainder results from lenghty but fairly simple calculations. ${ }^{11}$

We now turn to the proof of proposition 2.3. The assumptions make it possible to apply directly the stationary phase lemma. For a given $\nu$, the stationary points are solutions of $\varphi^{\prime}\left(t_{s}(\nu)\right)=2 \pi \nu$. The monotonicity of $\varphi^{\prime}$ and the considered domain of values of $\nu$ ensure existence and uniqueness of $t_{s}(\nu)$. The stationary phase method yields precisely the expression (2.10). For the remainder $r$, we have the following bound

$$
|r(\nu)| \leq \frac{5 \sqrt{\pi}}{4}\|B[f, a]\|_{\infty}, \text { where } f(t)=\varphi(t)-2 \pi \nu t .
$$

Controlling explicitely such bounds turns out to be quite difficult in practice. For that reasons, one sometimes uses the evaluation of $B(f, a)$ at the stationary point $t_{s}$ instead of its sup norm to estimate the order of magnitude of the remainder. 
Consider now the special case of power law chirps. In such a situation, the stationary point is explicitely given by

$$
t_{s}(\nu)=\left(\frac{\nu}{F_{0}}\right)^{1 / \beta}
$$

and the leading term in Corollary 2.1 follows immediately.

As mentioned above, an effective check of the validity of such approximations is generally problematic, since it requires the evaluation of a sup norm. A substitute amounts to replace the sup norm by the evaluation at the stationary point (i.e. the evaluation of the next term in the asymptotic expansion). Letting $I_{1}$ denote the estimation of the $\hat{x}$ provided by the stationary phase approximation, and $I_{2}$ the next nonzero term, it may be shown that

$$
\left|\frac{I_{2}}{I_{1}}\right| \approx \frac{C_{\alpha, \beta}}{F}\left(\frac{\nu}{F}\right)^{-(\beta+1) / \beta} .
$$

We notice that the bound is uniform with respect to $\nu$ for $\beta=-1$, namely for hyperbolically frequency modulated signals. We also notice that for the case of gravity waves we consider as target application in this paper, we obtain a behavior of the type $I_{2} / I_{1} \simeq \nu^{5 / 3}$. Hence the validity domain of the approximation lies in the low frequency region. This result has been obtained previously by ChassandeMottin and Flandrin ${ }^{4,5}$.

\section{Appendix B. Oscillatory integral approximations of continuous wavelet transform: proof of Proposition 3.1}

Let $\psi$ denote the Morlet wavelet, and consider the following oscillatory integral:

$$
\begin{aligned}
T_{x}(b, s) & =\frac{1}{\sqrt{s}} \int_{-\infty}^{\infty} a(t) e^{-(t-b)^{2} / 2 \sigma^{2} s^{2}} e^{i\left(\varphi(t)-\omega_{0}(t-b) / s\right.} d t \\
& =\frac{1}{\sqrt{s}} \int_{-\infty}^{\infty} a(t) e^{i \Phi(t)-(t-b)^{2} / 2 \sigma^{2} s^{2}} d t .
\end{aligned}
$$

Letting $t_{s}$ denote as usual a stationary point of $\Phi$, and setting $\beta=\Phi^{\prime \prime}\left(t_{s}\right)+i / \sigma^{2} s^{2}$ and

$$
u(t)^{2}=\frac{\Phi(t)-\Phi\left(t_{s}\right)+i\left[(t-b)^{2}-\left(t_{s}-b\right)^{2}\right] / 2 \sigma^{2} s^{2}}{\beta}
$$

we are led to an integral similar to the one considered in Lemma A.1 (the imaginary part of $\beta$ is indeed positive.) An argument similar to the stationary phase argument yields the result.

\section{References}

1. A. Abramovici et al., LIGO: the Laser Interferometer Gravitational-Wave Observatory, Science, Vol. 256, pp. 325-333 (1992).

2. C. Bradaschia et al. The Virgo project : a wide band antenna for gravitational wave detection, Nucl. Instrum. Methods Phys. Res. Vol. A289, pp. 518-525 (1990). 
3. R. Carmona, W. Hwang and B. Torrésani, Practical Time-Frequency Analysis. Gabor and Wavelet Transforms with an Implementation in $S$ (Academic Press, 1998).

4. E. Chassande-Mottin, Méthodes de réallocation dans le plan temps-fréquence pour l'analyse et le traitement de signaux non-stationnaires, $\mathrm{PhD}$ Thesis (Université de Cergy-Pontoise, 1998).

5. E. Chassande-Mottin and P. Flandrin, On the time-frequency detection of chirps, (Appl. Comp. Harm. Anal., Vol. 6, pp. 252-281 (1998).

6. I. Daubechies, Ten Lectures on Wavelets CBMS-NFS Regional Series in Applied Mathematics 61, (SIAM, 1992).

7. L. Daudet, M. Morvidone and B. Torrésani, Time-frequency and time-scale vector fields for deforming time-frequency and time-scale representations, Proceedings of the SPIE conference, Denver, M. Unser Ed., pp. 2-15. (1999).

8. N. Delprat, B. Escudié, P. Guillemain, R. Kronland-Martinet, Ph. Tchamitchian and B. Torrésani, Asymptotic wavelet and Gabor analysis: extraction of instantaneous frequencies, IEEE Trans. Inf. Th., Vol. 38, pp. 644-664 (1992).

9. P. Flandrin, Temps-Fréquence, Traité des Nouvelles Technologies, série Traitement du Signal, (Hermès, 1993).

10. A. Grossmann, J. Morlet, Decomposition of Hardy functions into square integrable wavelets of constant shape, SIAM J. of Math. An., Vol. 15, pp. 723 (1984).

11. J. Harthong, Etudes sur la mécanique quantique (Astérisque, 1984).

12. J.M. Innocent and B. Torrésani, Wavelets and Binary Coalescence Detection, Applied and Computational Harmonic Analysis, Vol. 4, pp. 113-116 (1997).

13. C. Hory, N. Martin and A. Chehikian, Spectrogram segmentation by means of statistical features for non-stationary signal interpretation, preprint (2001), to appear in IEEE Trans. Signal Processing.

14. K. Kodera, R. Gendrin, and C. de Villedary, Analysis of time-varying signals with small BT values, IEEE Trans. ASSP Vol. 26, p. 64 (1978).

15. L.H. Koopmans, The spectral Analysis of Time Series. Probability and Mathematical Statistics, Vol. 22 (Academic Press, 1995).

16. J. Lamperti, Stochastic Processes (Springer-Verlag, 1977).

17. S. Mallat, A Wavelet Tour of Signal Processing, (Academic Press, 1998).

18. Y. Meyer, Oscillating patterns in image processing and in some nonlinear evolution equations, the Fifteenth Dean Jaqueline B. Lewis Memorial Lectures, Rutgers University (2001).

19. M. Morvidone, Détection temps-fréquence, application à la détection des ondes gravitationnelles $\mathrm{PhD}$ Thesis, Marseille (2002).

20. M. Morvidone and B. Torrésani, Time-scale chirp detection and estimation: Application to gravity waves detection, Proceedings of the PSIP conference, Marseille, France pp. 264-269 (2001),

21. B. Picinbono and W. Martin, Représentation des signaux par amplitude et phase instantanées, Annales des Télécommunications, Vol. 38 , pp. 179-190 (1983).

22. Ph. Tchamitchian and B. Torrésani, Ridge and Skeleton extraction from wavelet transform, in Wavelets and their Applications, M.B. Ruskai \& Al Eds, (Jones\&Bartlett, 1991).

23. K. Thorne, Gravitational waves, Proceedings of the Snowmass'94 Summer Study on Particle and Nuclear Astrophysics and Cosmology, eds. E. W. Kolb and R. Peccei, pp.398-425. (World Scientific, 1995).

24. H. Van-Trees, Detection, Estimation, and Modulation Theory. Part I, (John Wiley \& Sons, 1968). 